\title{
Propuesta metodológica de priorización de áreas para conservación de cuencas. Validación en río Caquinal, Fómeque, Cundinamarca, Colombia.
}

\section{Methodological proposal of prioritizing of areas for conservation of hydrographic basins. Validation in the river Caquinal, Fómeque, Cundinamarca, Colombia}

\section{Proposta metodológica para priorização de áreas para a conservação de bacias hidrográficas. Validação de rio Caquinal, Fómeque, Cundinamarca, Colômbia.}

\author{
Álvaro Martín Gutiérrez Malaxechebarría1, Yorlenny Zambrano Rodríguez² \& Laura Ospina Hoyos ${ }^{3}$ \\ IIngeniero Civil, Magíster en Ingeniería Civil, Doctor en Estudios Ambientales y Rurales. ${ }^{2,}{ }^{3}$ Estudian- \\ te de Ingeniería Ambiental de la Universidad Distrital Francisco José de Caldas. Bogotá. Colombia. \\ 1, 2,3Proyecto Curricular Ingeniería Ambiental. Facultad de Medio Ambiente y Recursos Naturales. \\ Universidad Distrital Francisco José de Caldas. Bogotá. Colombia. \\ 19amgutierrezm@udistrital.edu.co, 2yors06@hotmail.com, ${ }^{3}$ lauospinah0524@gmail.com
}

\section{Resumen}

Las cuencas hidrográficas son unidades de territorio que suplen las necesidades humanas a través de la oferta de recursos naturales, sin embargo, estas áreas también deben ser consideradas espacios potenciales de conservación y garantía de recursos para las generaciones venideras. Esta investigación plantea una propuesta de priorización de áreas estratégicas de conservación para la regulación de caudales, validada con el apoyo de la comunidad en la microcuenca del río Caquinal ubicada en el municipio de Fómeque, Cundinamarca, Colombia. La propuesta incluye elementos de índole físico-ambiental, normativo, socioeconómico y cultural, enfocados a integrar y a entender la dinámica de la cuenca hidrográfica, en cuanto a su respuesta hidrológica dentro del modelo lluvia-escorrentía, su relación con la morfometría, los múltiples usos del recurso hídrico y los lineamientos de orden ambiental, legislativo y social que aplican a una zona de estudio. La metodología, está dividida en dos fases, la primera corresponde al análisis de la cuenca y la segunda específicamente a la priorización de áreas, en la cual se propone la aplicación de la matriz descriptiva que reúne las principales variables a analizar de cada área y permite identificar cuáles de éstas son prioritarias o estratégicas. Se encontró que la metodología desarrollada constituye una herramienta para la toma de decisiones en la protección de cuencas hidrográficas y se espera que guie las acciones para priorizar áreas con diversos fines y objetivos.

Palabras clave: áreas estratégicas, comunidad, conservación, cuenca hidrográfica, recurso hídrico. 


\section{Abstract}

The hydrographic basins are units of territory that meet human needs through the supply of natural resources, however, these areas should also be considered potential spaces of conservation and guarantee of resources for future generations. This research presents a proposal for prioritization of strategic areas of conservation for the regulation of flows, validated with the support of the community in the watershed of the Caquinal River, located in the municipality of Fómeque, Cundinamarca, Colombia. The proposal includes elements of nature physical-environmental, regulatory, socioeconomic and cultural, aimed to integrate and understand the dynamics of the river basin, in terms of their hydrological response within the model rain-runoff, his relationship with morphometry, the multiple uses of water resources and guidelines for environmental, legislative and social order that applied to a study area. The methodology, is divided into two phases, the first corresponds to the analysis of the basin and the second specifically on the prioritization of areas, which proposes the application of the descriptive matrix that brings together the main variables to analyze in each area and it identifies which of these areas are priorities or strategic. It was found that the methodology developed is a tool for decision making at watershed protection and it is expected to guide the actions to prioritize areas for different purposes and objectives.

Key-words: strategic areas, community, conservation, hydrographic basin, water resource

\section{Resumo}

As bacias hidrográficas são unidades do territorio que atendem as necesidades humanas através da oferta de recursos naturais, no entanto essas áreas debem se- considerar espaços potenciais de conservação e garantía de recursos naturais para para as gerações futuras. Esta pesquisa apresenta uma proposta de priorização de áreas estratégicas de conservação para a regulação das cidades, validada com apoio da comunidade na microbacia do rio Caquinal localizado no município de Fómeque, Cundinamarca, Colômbia. A proposta inclui elementos de natureza físico-ambiental, regulatória, socioeconômica e cultural, visando a integração e compreensão da dinâmica da bacia do rio, em termos de resposta hidrológica dentro do modelo chuva-escoamento, sua relação com a morfometria, os múltiplos usos dos recursos hídricos e diretrizes para a ordem ambiental, jurídica e social aplicada a uma área de estudo. A metodologia é dividido em duas fases, a primeira é a análise da bacia e a segunda fase, especificamente prioridade áreas em que se propõe a aplicação de matriz descritiva, que inclui. As principais variáveis a serem analisadas em cada área e identificar quais delas são estratégicos ou de prioridade. Verificou-se que a metodologia desenvolvida, é uma ferramenta para tomada de decisão na proteção de bacias hidrográficas e é esperado para orientar as ações para priorizar áreas para diversos fins e objetivos.

Palavras-chave: áreas estratégicas, comunidade, conservação, bacias hidrográficas, recurso hídrico.

\section{Introducción}

Las cuencas hidrográficas son unidades de territorio que funcionan mediante la interacción de un subsistema hídrico que produce agua, junto con un subsistema económico y social, activado por el hombre, el capital, el trabajo y la tecnología (Díaz Granados \& Gutiérrez, 2007). Si bien, las cuencas hidrográficas se delimitan mediante una divisoria de aguas, que es una línea imaginaria que separa la superficie de tierra cuyo drenaje fluye hacia un cauce dado, de las superficies de tierra cuyos drenajes corren hacia otro cauce (Chow, 1994); debido al uso que hacen las diferentes poblaciones sobre estas, también se debe considerar una delimitación que refleje la demanda real de los 
recursos de la cuenca, pues no siempre los usuarios que hacen uso de estos recursos se localizan dentro del límite natural de está. Así, el área total sobre la cual se ubican los diferentes usos humanos del agua de una cuenca se ha denominado cuenca social. Este concepto expresa el área total sobre la cual una población hace uso de los recursos naturales de una cuenca incluso más allá de sus límites hidrológicos.

Es necesario buscar alternativas de conservación y protección de los recursos, donde a la vez que se pueda hacer aprovechamiento de los recursos se garantice también su accesibilidad para las generaciones venideras, mediante estrategias que involucren alternativas más allá de los límites naturales y logren una adecuada mejoría de las áreas de una cuenca, considerando los usos que se dan al recurso hídrico, como bien común y como eje de participación social de las comunidades beneficiarias. Para ello se ha formulado una metodología que pretende favorecer la regulación de los caudales de la cuenca, manteniendo a través del tiempo la dotación de recurso hídrico, y así fortalecer la respuesta del sistema de la cuenca frente a alguna eventualidad; considerando de vital importancia el cuidado del tejido hídrico principal como lo son nacimientos de agua, pantanos, manantiales, rondas hídricas, entre otros; así como la inclusión de la comunidad en los procesos y proyectos que se desarrollen.

Esta investigación tiene como objetivo aplicar dicha metodología a la priorización de áreas para la conservación de una cuenca que abastece a una comunidad rural en los Andes Colombianos la cual otorga múltiples usos al recurso hídrico; resaltando que se contó con el apoyo de dicha comunidad. La iniciativa de seleccionar áreas que permitan mantener el ecosistema, las relaciones sociales y la regulación natural de los caudales, considera como aspectos fundamentales para alcanzar una conservación eficaz: definir, conocer y dar prioridad a los lugares en los que se tiene que actuar en primera instancia (Newton, 2011), es decir, la identificación de áreas estratégicas que propicien la dinámica hidrológica y la respuesta natural de la cuenca a los fenómenos de precipitación-evaporación-infiltración.

En esta investigación fueron considerados elementos de carácter físico-ambientales, normativos, socioeconómicos y culturales; enfocados a integrar y a entender la dinámica de la cuenca hidrográfica, en cuanto a su respuesta hidrológica dentro del modelo lluvia-escorrentía, su relación con su morfometría, los múltiples usos del recurso hídrico y los lineamientos de orden ambiental, legislativo y social que aplican a la zona de estudio.

Dentro de los factores que afectan la respuesta hidrológica de una cuenca se han clasificado 5 grupos así: factores morfométricos, factores climáticos, factores asociados al suelo, factores de cobertura vegetal y los factores sociales. Estos factores están relacionados con la descripción física de la cuenca, su comportamiento ante un fenómeno de precipitación, los regímenes de caudales, el desarrollo del suelo, el tipo de vegetación, los procesos de infiltración y percolación en el suelo dada su conductividad hidráulica para diferentes condiciones de humedad, entre otros.

Si bien, la regulación de caudales está fuertemente relacionada con las variables de orden natural, también depende de las características sociales que se den en la zona de estudio, ya que los diferentes usos del agua y el suelo, están condicionados a las actividades económicas y culturales de la población. Además, el suelo está relacionado con las coberturas vegetales presentes en la cuenca, cómo éstas contribuyen a la regulación hídrica y evidencian el grado de alteración o intervención humana a las diferentes áreas (Deeb, 1992). Debido a esto, es necesario considerar los aspectos sociales y culturales como permanentes modeladores de la dinámica de una cuenca, entendiendo además que su comportamiento está ligado a una memoria cultural y tradiciones propias de cada región. 


\section{Metodología}

\section{Contexto}

La presente investigación se desarrolló en la microcuenca del río Caquinal, ubicada en el noroccidente del municipio de Fómeque, Cundinamarca, Colombia, con un área natural de 2316 ha y una cuenca social de 4992 ha, abas- teciendo a la población de 7 veredas del municipio y al Centro Poblado La Unión, con un total aproximado de 900 usuarios. Según la Corporación Autónoma Regional del Guavio CORPOGUAVIO, la microcuenca está en agotamiento, es decir que está imposibilitada par a soportar nuevos usos. (CORPOGUAVIO, 2011). En la Figura 1 , se visualiza el área y la ubicación de la zona de estudio.

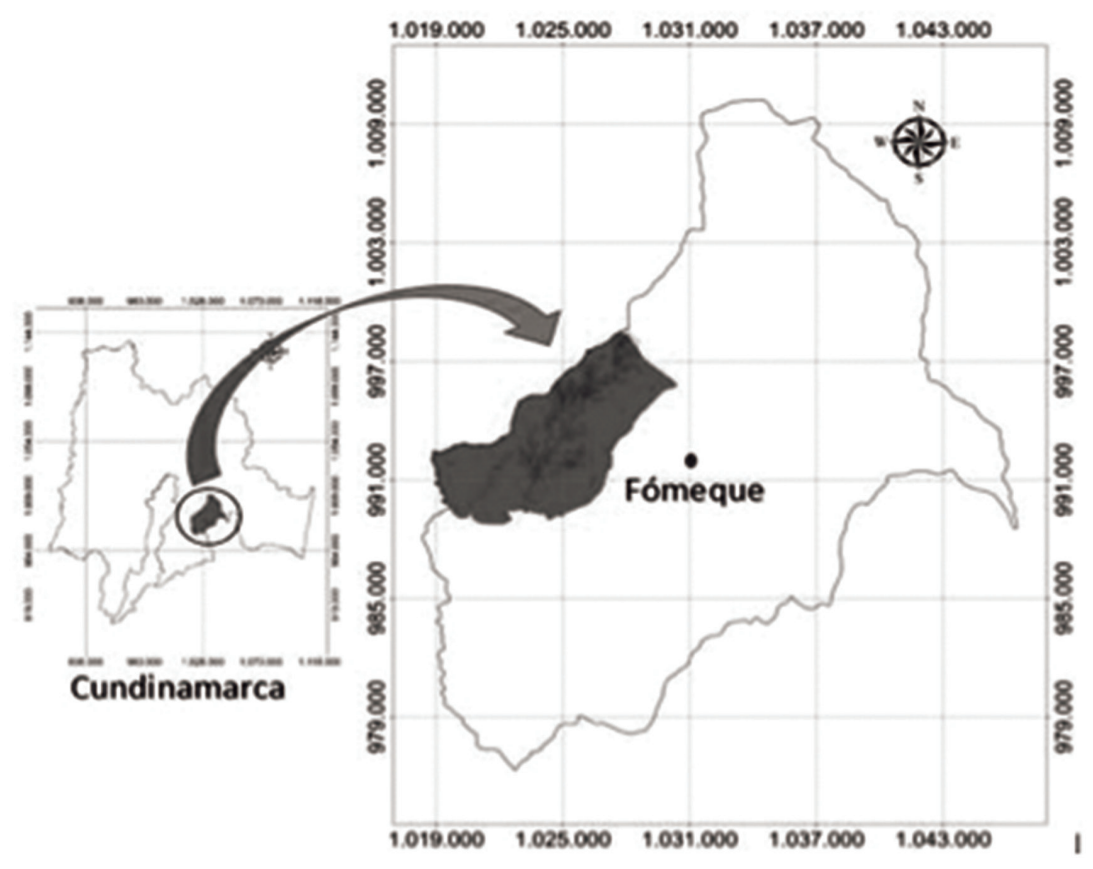

Figura 1. Área y ubicación de la zona de estudio.

\section{Características físico-ambientales}

El río Caquinal nace en el páramo de Chingaza a 3370 msnm, en el humedal El Diamante, nutriéndose más adelante de los ríos Blanca y La Chorrera, hasta llegar al río Negro que se prolonga hasta el Orinoco con el nombre de río Guayuriba. La cuenca social presenta alturas que oscilan entre los 1700 y 3660 msnm, lo cual permite la presencia de varios pisos térmicos registrando en esta zona temperaturas medias que oscilan entre los $9^{\circ} \mathrm{C}$ y $21^{\circ} \mathrm{C}$. Estos factores climatológicos y topográficos favorecen la presencia de las zonas de vida: Sub-páramo, Bosque Alto Andino, Bosque Andino y Bosque Sub-andino; se presentan pendientes que van desde ligeramente planas hasta totalmente escarpadas. El régimen de lluvias es de tipo monomodal, con precipitaciones medias en la parte alta de $1675 \mathrm{~mm}$ anuales y en la parte baja de $975 \mathrm{~mm}$ anuales, con un periodo de mayor precipitación en los últimos meses del año (Universidad Nacional de Colombia, 2000). En cuanto a la geología, las formaciones presentes son rocas sedimentarias compuestas por bancos potentes de lodolitas negras y arcillolitas café con abundante pirita. La geomorfología se caracteriza por ser un paisaje de montaña con relieve de origen estructural-denudacional y agradacional. La cuenca presenta 12 unidades cartográficas de suelos, en su mayoría con textura Franco-Arenosa. 


\section{Características socioeconómicas}

Las cuencas alta, media y baja, se definieron de acuerdo a los siguientes criterios: la cuenca alta es aquella que va desde el nacimiento del río Caquinal hasta el punto en que se encuentra la última bocatoma a $2531 \mathrm{msnm}$, la cuenca media de ahí hasta 2013 msmn y la cuenca baja hasta su desembocadura en el Rio Negro a 1735 msnm. La cuenca alta se encuentra fuertemente potrerizada por el efecto histórico de la deforestación y la instalación de la ganadería extensiva, tiene un área de 1788,69 ha y presenta una baja ocupación humana. En la cuenca media se ubica la mayor parte de la población rural y cultivos dirigidos al mercado, en especial, habichuela (Phaseolus vulgaris), tomate (Lycopersicum esculentum), pimentón (Capsicum annuum), cebolla (Allium cepa), pepino de rellenar (Cyclanthera Pedata), entre otros, así como a la actividad avícola y porcícola de mediana escala. En la cuenca baja se ubican industrias avícolas, multinacionales de producción de esquejes, fincas de recreo y empresas turísticas, así como el Centro poblado La Unión.

\section{Organización comunitaria de la cuenca social}

El río Caquinal abastece dos acueductos comunitarios y tres distritos de riego, los cuales se agruparon en el año 2010 en la Asociación de Segundo Grado de Usuarios de la Cuenca Caquinal ASOCAQUINAL cuyo "objeto principal es concertar el adecuado manejo de los recursos naturales y culturales de la cuenca de Caquinal, en cooperación con los distritos de riego y acueductos de ésta, así como con las entidades gubernamentales y no gubernamentales de orden nacional e internacional" (Estatutos ASOCAQUINAL, 2010). Además de los usuarios legalmente constituidos la río soporta numerosos usuarios de hecho, localizados de forma dispersa y no representados por ASOCAQUINAL, así como también un nuevo distrito de adecuación de tierras, denominado ASORENACER que tampoco está incluido en dicha asociación. En la Figura 2 se evidencian las diferentes organizaciones sociales que tienen concesión de aguas sobre el río Caquinal y abastecen a un cierto número de usuarios con un caudal establecido y regulado por CORPOGUAVIO.

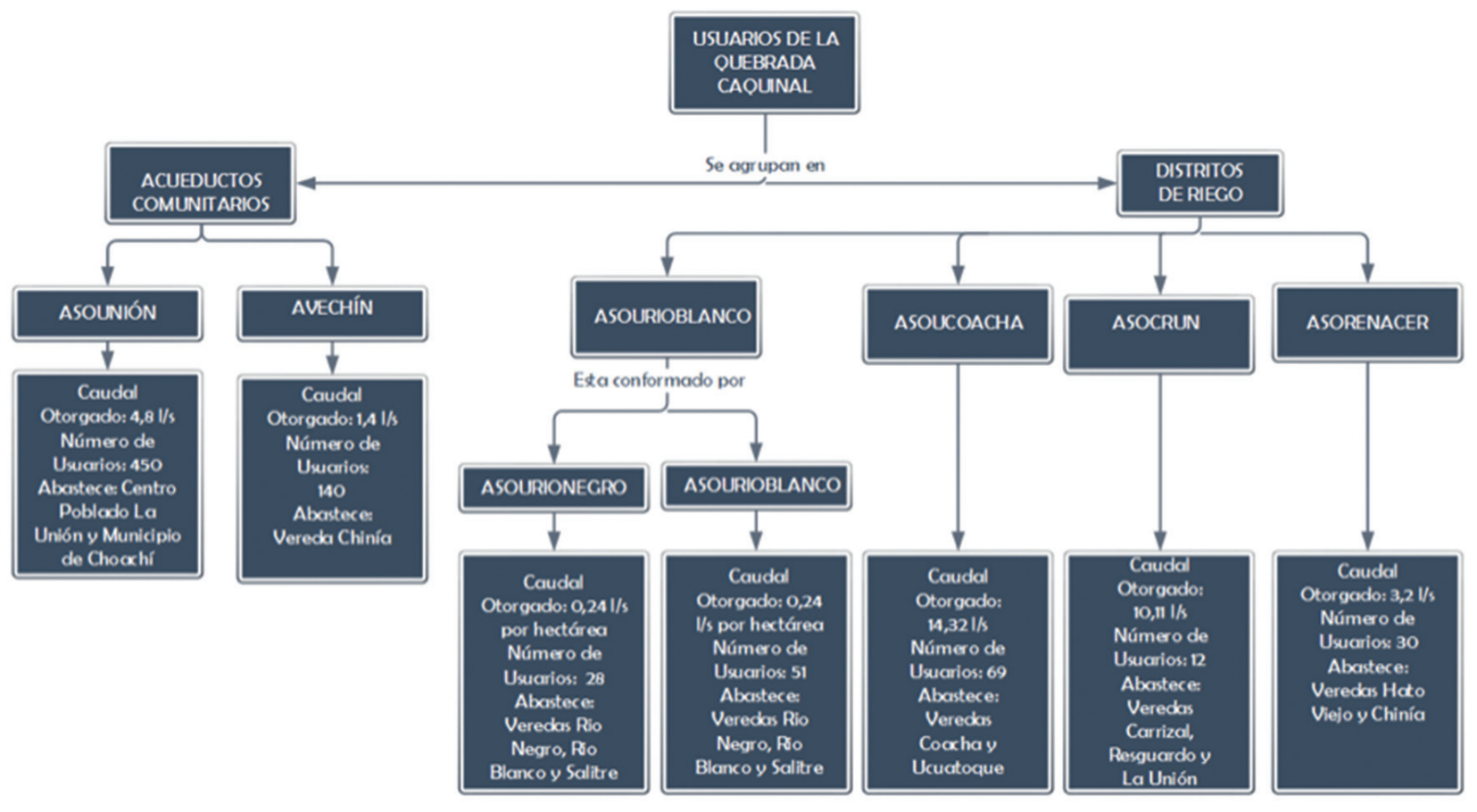

Figura 2. Esquema de los usuarios del Río Caquinal. 


\section{Fases metodológicas}

La metodología, está dividida en dos fases, la primera corresponde al análisis de la cuenca y la segunda específicamente a la metodología para la priorización de áreas.

\section{Fase I: Análisis de la cuenca}

\section{Búsqueda y recopilación de información}

Esta recopilación de datos se realizó en dos etapas: la primera fue la consulta de la información secundaria. En la segunda etapa se llevó a cabo el acercamiento con la comunidad y el levantamiento de información primaria en campo, de los diferentes datos relacionados con la estructura social de la cuenca, actividades económicas, cambios de uso del suelo y usos del recurso hídrico. Dentro de la información secundaria que se consultó, se tomó en cuenta la cartografía de la zona de estudio, que se digitalizó y procesó utilizando el programa ArcGis 10, así: Topografía, Hidrología y Pendientes (IGAC, 1995); Geología (Servicio Geológico Colombiano (SGC), 2000); Suelos (IGAC, 2000); Coberturas Vegetales en Lansat Imagery de ArcGis 10 (ESRI , 2010) clasificadas teniendo en cuenta las coberturas establecidas en la Geodatabase de la Agencia Nacional De Licencias Ambientales (ANLA), utilizadas para la generación de cartografía de proyectos. Climática: estación climatológica de temperatura y precipitación del Instituto de Hidrología, Meteorología y Estudios Ambientales de Colombia (IDEAM) conocida como Estación Fómeque y las Estaciones Hato Viejo, El Paval, La Unión, Laguna Chingaza y La Playa de la Empresa de Acueducto y Alcantarillado de Bogotá (EAAB). Político-administrativo (Gobernación de Cundinamarca, 2000). Información Predial: subdirección de catastro del IGAC. Erosionabilidad: aplicación de las formulas de la ley de
Darcy y Factor K de erosionabilidad (Zuñiga Palma, 2010), junto con la información de las unidades del suelo (IGAC, 2000). Erodabilidad: superponiendo la información de erosionabilidad, precipitación y pendientes, (Zuñiga Palma, 2008). Amenazas y Riesgos: cartografía temática de amenazas por avalanchas, remoción en masa, inundaciones e incendios (CORPOGUAVIO, 2005), corroborándose con las visitas hechas en campo.

\section{Identificación de actores y organizaciones comunitarias en la cuenca}

La identificación de actores se realiza a través del acercamiento a la comunidad en las visitas de campo, hablando sobre la gestión comunitaria del agua, la cual se ha dado a lo largo de los años con los presidentes de los distritos de riesgo y con los usuarios, quienes viven en las diferentes partes de la cuenca; también se indaga sobre la relación de los residentes y trabajadores de la cuenca alta con el recurso hídrico y su accesibilidad a éste. Además se leyó la información que poseen las diferentes entidades encargadas del manejo del recurso hídrico como Io son CORPOGUAVIO y ASOCAQUINAL. Esta identificación de actores permite definir la cuenca social y la cantidad de usuarios que demandan agua de la río.

\section{Aplicación de encuestas}

Dentro de las estrategias utilizadas para recopilar información del área de estudio, se realizaron una serie de encuestas que tomaban información sobre las percepciones frente a temas relacionados con el medio ambiente, memoria cultural e importancia de priorización de áreas en la cuenca alta, para lo cual fue necesario realizar un formulario con 33 preguntas, unas con repuesta abierta, otras con respuesta Si/No y otras con opciones; 
que indagan sobre la relación de la comunidad con el recurso hídrico y la necesidad de este recurso para el desarrollo de las diferentes actividades económicas. Así como también, indagar sobre la idea que se tiene de la protección del recurso, el ahorro de éste y las medidas de conservación que se pueden realizar en la cuenca. El tamaño poblacional de la muestra fue de 66 personas, se tomó por muestreo en bola de nieve, propuesto por Goodman (1961).

\section{Características de la cuenca en relación con la respuesta hidrológica}

Se consideraron los parámetros morfométricos que condicionan la respuesta de la cuenca a los fenómenos lluvia-escorrentía y se calcularon los parámetros más relevantes para precisar las características físicas de la cuenca, como se describe a continuación:

Pendiente promedio de la cuenca $(\mathrm{Im})^{2}$, se calcula con base en el mapa topográfico a escala 1:10.000, a partir del cual se genera un modelo digital de terreno y posteriormente un mapa de pendientes. Del cual se obtienen porcentajes de área por cada rango de pendiente y a partir de esta información se realiza un análisis estadístico donde se obtiene finalmente la pendiente promedio de la cuenca utilizando Sistemas de Información Geográfica como lo es el software Arc GIS 10 proveído por (ESRI , 2010).

Pendiente del cauce principal (Ic). Los parámetros de forma de las cuencas normalmente están asociados a relaciones del área, la longitud del cauce mayor y medidas de "esfericidad". Dentro de los índices a analizar en este caso, se consideraron:
Factor de Forma de Horton (kf), se obtiene a partir de la siguiente relación (Horton, 1932):

$$
k f=\frac{\text { Área de cuenca }}{\text { Longitud de la cuenca }{ }^{2}}
$$

Coeficiente de Compacidad o índice de Gravelius ( $k c)$, se calculó a partir de la siguiente formula (Gravelius, 1941):

$$
k c=\frac{\text { Perimetro de la cuenca }}{2 * \sqrt{\pi * \text { Área }}}
$$

Índice de alargamiento (la), se calculó mediante la siguiente formula, propuesto por (Horton, 1945):

$$
I a=\frac{\text { Longitud Máxima daela Cuenca }}{\text { Ancho Máximo de la Cuenca }}
$$

\section{Fase II Metodología para la priorización de áreas}

Esta fase de la metodología, se lleva a cabo a través de la construcción de una propuesta de identificación de áreas estratégicas y vitales para la regulación hídrica, que permitan regular los caudales del río y garanticen el acceso al recurso hídrico; esta metodología se fundamenta en 3 aspectos:

\section{a. Definición de variables}

Es necesario identificar qué variables se tendrán en consideración, para ser evaluadas y de esta forma conocer las diferentes escalas de análisis que tendrá cada una en el sentido de su aporte a la identificación de las áreas estratégicas. Estas variables son de orden físico-ambiental, normativo, socioeconómico y cultural, como se observa en la Tabla 1. 
Tabla 1. Variables a considerar en la metodología de priorización de áreas.

\section{VARIABLES FISICO-AMBIENTALES}

\begin{tabular}{ll}
\hline & Geología \\
& Geomorfología \\
& Paisaje \\
& Pendientes \\
& Erosionabilidad \\
GEOSFÉRICAS & Erodabilidad \\
& Asociación de Suelos \\
& Clases Agrologicas \\
& Humedad del suelo \\
& Granulometría del suelo \\
& Amenazas y Riesgos \\
& Precipitación \\
CLIMATOLÓGICAS & Evapotranspiración \\
& Temperatura \\
FLORA & Coberturas Vegetales \\
FAUNA & Especies Nativas \\
& Rondas Hídricas \\
HIDROLÓGICAS & Pantanos \\
& Nacimientos de agua
\end{tabular}

\section{VARIABLES NORMATIVAS}

Parques Nacionales Naturales

Parques Naturales Regionales

Presencia de páramos y sub-páramos

ÁREAS PROTEGIDAS Y ÁREAS DE ESPECIAL IMPORTANCIA ECOSISTÉMICA

Distritos de Manejo Integrados

Distritos de conservación de suelos

Áreas de recreación

Reservas Naturales de la Sociedad Civil

Otras áreas protegidas por otras entidades.

\section{VARIABLES SOCIOECONÓMICAS}

USO DEL SUELO

Actividad Económica

Vías de acceso

INFRAESTRUCTURA

Asentamientos

Equipamientos

\section{VARIABLES CULTURALES}

Sitios de Interés

Sitios de memoria histórica

IMPORTANCIA CULTURAL

Sitio Arqueológico

Áreas de valor social

Lugares de referencia/ubicación 
b. Levantamiento de datos en campo

Para confirmar los puntos de relevancia y la localización de los predios fue necesario visitar cada uno de los predios de la cuenca alta y hacer el respectivo levantamiento del Sistema de Posicionamiento Global (GPS) de las diferentes coberturas vegetales encontradas, así como elementos asociados al recurso hídrico entre los que se pueden encontrar pantanos, nacimientos, afluentes secundarias, ubicación de las bocatomas, desarenadores entre otros. Esta información se carga al software ArcGis 10 (ESRI , 2010), donde se visualizan los puntos tomados con el GPS.

c. Interpretación y evaluación de la información

Se evalúan las variables en la matriz que relaciona unidades administrativas en este caso, predios, con las variables en consideración. Resaltando las características más influyentes en la regulación hídrica, como lo son: ausencia y presencia de cuerpos hídricos, tipo de suelo, coberturas vegetales, la importancia cultural entre otros. Entre más variables asociadas a la regulación de caudales se presenten en un predio, más importante resulta ser éste, ya que la representatividad de su conservación aumenta. Cuando la metodología se utilice para otros fines deberá establecerse qué variables son más relevantes para definir la escala de alta, media y baja importancia.

En la Tabla 2 se expone un ejemplo de la matriz que se formuló para sintetizar e integrar la información obtenida en la presente investigación.

Tabla 2. Matriz Descriptiva de la información.

\begin{tabular}{|c|c|c|c|c|}
\hline \multirow{2}{*}{\multicolumn{2}{|c|}{$\begin{array}{l}\text { VARIABLES FÍSICO- } \\
\text { AMBIENTALES }\end{array}$}} & \multicolumn{3}{|c|}{ ÁREAS } \\
\hline & & A & B & C \\
\hline \multirow{11}{*}{$\begin{array}{l}\text { GEOSFÉ- } \\
\text { RICAS }\end{array}$} & Geología & Formación Fómeque & Formación Fómeque & $\begin{array}{l}\text { Formación } \\
\text { Fómeque }\end{array}$ \\
\hline & Geomorfología & $\begin{array}{l}\text { Espinazos, Crestas, } \\
\text { Escarpes, Barras } \\
\text { Homoclinales, Gla- } \\
\text { cis Coluvial, lomas }\end{array}$ & Glacis Coluvial & Crestones \\
\hline & Paisaje & Media Montaña & Media Montaña & Media Montaña \\
\hline & Pendientes & $50-75 \% ; 12-25 \%$ & $12-25 \%$ & $25-50 \%$ \\
\hline & Erosionabilidad & Media & Media & Media \\
\hline & Erodabilidad & Media & Media & Media \\
\hline & Asociación de Suelos & MGFf- MLKd-MGTd & MLKd & MLVe \\
\hline & Clases Agrologicas & VII -IV - VI & IV & VI \\
\hline & Humedad del suelo & $\begin{array}{l}\text { Muy Húmedo- Hú- } \\
\text { medo a transicional } \\
\text { seco }\end{array}$ & $\begin{array}{l}\text { Húmedo a transicio- } \\
\text { nal seco }\end{array}$ & $\begin{array}{l}\text { Húmedo } \\
\text { transicional seco }\end{array}$ \\
\hline & Granulometría del suelo & Franco Arenoso & Franco Arenoso & Franco arcillosa \\
\hline & Amenazas y Riesgos & $\begin{array}{l}\text { Fenómenos de } \\
\text { remoción en masa } \\
\text { y erosión hídrica } \\
\text { ligera }\end{array}$ & $\begin{array}{l}\text { Fenómenos de } \\
\text { remoción en masa }\end{array}$ & \\
\hline
\end{tabular}


...continuación Tabla 2

\begin{tabular}{|c|c|c|c|c|}
\hline \multirow{2}{*}{\multicolumn{2}{|c|}{$\begin{array}{l}\text { VARIABLES FÍSICO- } \\
\text { AMBIENTALES }\end{array}$}} & \multicolumn{3}{|c|}{ ÁREAS } \\
\hline & & A & B & C \\
\hline \multirow{3}{*}{$\begin{array}{l}\text { CLIMATO- } \\
\text { LOGICAS }\end{array}$} & Precipitación & $1300-1950 \mathrm{~mm}$ & $1300-1950 \mathrm{~mm}$ & $1300-1950 \mathrm{~mm}$ \\
\hline & Evapotranspiración & & & \\
\hline & Temperatura & $9-12^{\circ} \mathrm{C}$ & $12-15^{\circ} \mathrm{C}$ & $15-18^{\circ} \mathrm{C}$ \\
\hline FLORA & Coberturas Vegetales & $\begin{array}{l}\text { Bosque abierto, ar- } \\
\text { bustal y pasto limpio }\end{array}$ & pastos limpios & $\begin{array}{l}\text { Arbustal y pastos } \\
\text { limpios }\end{array}$ \\
\hline FAUNA & Especies Nativas & Sin registro & Sin registro & Sin registro \\
\hline \multirow{3}{*}{$\begin{array}{l}\text { HIDROLÓ- } \\
\text { GICAS }\end{array}$} & Rondas Hídricas & & & \\
\hline & Pantanos & & & \\
\hline & Nacimientos de agua & & & \\
\hline \multicolumn{2}{|c|}{ VARIABLES NORMATIVAS } & A & B & C \\
\hline
\end{tabular}

Parques Nacionales

Naturales

Parques Naturales

Regionales

ÁREAS Presencia de páramos

GIDASY y sub-páramos

ÁREAS DE Distritos de Manejo

ESPECIAL Integrados

IMPOR- Distritos de conserva-

TANCIA ción de suelos

EĆOSIS- Áreas de recreación

TEMICA

Reservas Naturales

de la Sociedad Civil

Otras áreas protegidas

por otras entidades

\begin{tabular}{|c|c|c|c|c|}
\hline \multicolumn{2}{|c|}{$\begin{array}{l}\text { VARIABLES SOCIO } \\
\text {-ECONÓMICAS }\end{array}$} & \multirow[b]{2}{*}{ Ganadería extensiva } & B & \multirow[b]{2}{*}{ Ganadería extensiva } \\
\hline $\begin{array}{l}\text { USO DEL } \\
\text { SUELO }\end{array}$ & Actividad Económica & & Ganadería extensiva & \\
\hline \multirow{3}{*}{$\begin{array}{l}\text { INFRAES- } \\
\text { TRUCTU- } \\
\text { RA }\end{array}$} & Vías de acceso & NO & NO & NO \\
\hline & Asentamientos & SI & NO & NO \\
\hline & Equipamientos & NO & NO & NO \\
\hline \multicolumn{2}{|c|}{ VARIABLES CULTURALES } & A & B & C \\
\hline \multirow{5}{*}{$\begin{array}{l}\text { IMPOR- } \\
\text { TANCIA } \\
\text { CULTURAL }\end{array}$} & Sitios de Interés & & & \\
\hline & $\begin{array}{l}\text { Sitios de memoria } \\
\text { histórica }\end{array}$ & & & \\
\hline & Sitio Arqueológico & & & \\
\hline & Áreas de valor social & & & \\
\hline & Lugares de referencia & & & \\
\hline
\end{tabular}

*El color gris indica presencia del elemento en consideración 


\section{Resultados}

\section{Cálculo de los parámetros morfométricos}

Estos cálculos permitieron conocer las características físicas de la cuenca y el comportamiento de los flujos del agua a lo largo y ancho de esta, de acuerdo con la topografía y la forma de la cuenca. Reconociendo que, estas características influyen en la respuesta hidrológica de la cuenca.

\section{Pendiente promedio de la cuenca (Im) ${ }^{2}$}

El porcentaje de área por cada rango de pendiente se muestra en la Tabla 3.

Tabla 3. Rango de Pendientes y su porcentaje de área en la cuenca.

\begin{tabular}{lccc}
\hline Pendiente (\%) & Área (\%) & Pendiente (\%) & Área (\%) \\
\hline $0-1$ & 0,27 & $25-50$ & 46,14 \\
$1-3$ & 0,39 & $50-75$ & 15,97 \\
$3-7$ & 2,01 & $75-100$ & 4,64 \\
$7-12$ & 4,48 & $>100$ & 1,24 \\
$12-25$ & 24,86 & & \\
\hline
\end{tabular}

Se estimó una pendiente media para la cuenca de $26,81 \%$, que corresponde a pendientes ligeramente escarpadas o ligeramente empinadas; esta característica provoca grandes velocidades en el desplazamiento del agua por los cauces induciendo erosión en estos, y por consecuente transporte de grandes cantidades de sedimentos de distintos tamaños, representados por el arrastre de partículas desde la cuenca alta hasta la cuenca baja en el punto de desembocadura del río Caquinal al rio Negro. Este índice está relacionado, también, con el área de posible inundación en crecidas y con los tiempos de concentración.

\section{Factor de Forma de Horton}

Para la cuenca del río Caquinal se obtiene un valor de 0,21. Debido a que es un valor menor que 1 y cercano a cero, indica que la cuenca es de forma rectangular y muy alargada.

Esto revela una tendencia a una menor amortiguación de las crecientes por efecto de la forma alargada de la cuenca, ya que permite que el agua fluya hacia la garganta de la cuenca en menos tiempo y sin que se generen empozamientos en la cuenca media y baja.

\section{Coeficiente de Compacidad o Índice de Gravellus}

Para la cuenca del río Caquinal se obtiene un vaIor de 1,57, el cual indica que la cuenca es de tipo oval redonda a rectangular oblonga, por tanto la cuenca presenta una susceptibilidad de grado menor a las crecidas.

\section{Índice de Alargamiento}

Para la cuenca del río Caquinal se obtiene un valor de 3,23, que indica que la cuenca es alargada; ya que esta presenta un área más larga que ancha.

\section{Propuesta metodológica de priorización de áreas}

Con la intención de construir una propuesta metodológica de priorización de áreas efectiva y ajustada a los objetivos del estudio, se sugiere seguir unos pasos que guíen las acciones y permitan la correcta aplicación de los esfuerzos técnicos y 
económicos, para lo cual se presentan a continuación los lineamientos sugeridos por los autores:

a. Defínase el cuerpo de agua principal y sus tributarios, así como su nacimiento y su desembocadura.

b. Identifíquese la cuenca hidrográfica con ayuda de las curvas de nivel del terreno y la divisoria de aguas para conocer el área de la cuenca natural.

c. Establézcase la cuenca alta, media y baja de acuerdo con las características del terreno y de la configuración de los drenajes, sabiendo que la cuenca alta se caracteriza por la densidad en los drenajes y el nacimiento de los cuerpos de agua; en la cuenca media la topografía del terreno cambia y se disminuye la densidad de los drenajes, quedando uno o dos cuerpos de agua principales que discurren hacia la parte baja, (a esta parte de la cuenca se le conoce como garganta); luego se encuentra el área de la cuenca baja, conocida también como cono de deyección, donde el cauce se conforma por un solo ramal de salida de agua o punto de descarga, además de esto el relieve también cambia con respecto a la cuenca alta y media, disminuyendo la pendiente o el grado de inclinación.

d. Habiendo identificado las tres partes de la cuenca es necesario decidir en qué zona se hará la priorización de áreas de acuerdo al objetivo del estudio.

e. Consúltese la información relacionada con la caracterización biofísica del área seleccionada de la cuenca, información de suelos, coberturas vegetales, geología, topografía, zonas de amenazas y riesgos, usos del suelo e infraestructura. (De ser posible esto debe realizarse en la búsqueda de información primaria y secundaria como fase inicial del estudio).

f. Defínase una unidad de análisis para llevar a cabo la priorización de áreas.
*De ser la unidad predial, la unidad de priorización de áreas es necesario sobreponer los límites prediales a la información de la cuenca.

${ }^{* *}$ De no ser así, se debe establecer una unidad sobre la que se vaya a realizar la priorización, bien sea por tipos de suelo, pendientes, coberturas vegetales, entre otros y sobreponer dicha unidad de análisis al área de la cuenca para generar los diferentes polígonos.

g. Identifíquense los actores sociales involucrados en la cuenca hidrográfica y su relación con esta, en el sentido del uso y aprovechamiento de los recursos para la producción de bienes o servicios, que constituyan el frente socioeconómico de la zona (Restrepo, 2005). Será necesario hacer un acercamiento a los líderes comunitarios y a la comunidad en general, consultando e indagando la información social que pueda ser útil para el desarrollo del proyecto. (La información social recolectada será una variable de juicio y evaluación a tener en cuenta dentro del estudio).

h. Conociendo la mayor cantidad de características que describan la zona de estudio, es preciso iniciar con los parámetros que constituyen la base de priorización de las áreas, así:

- Identificar los predios que pertenezcan a Parques Nacionales Naturales, Parques Naturales Regionales, Distritos de Conservación de Suelos, Distritos de Manejo Integrado, Reservas de la Sociedad Civil o las áreas protegidas por otras entidades territoriales.

- Establecer los nacimientos de agua y los contribuyentes al cuerpo de agua principal.

Crear una zona de amortiguación de 100 metros a la redonda en los nacimientos de agua (Dostal, 2007). A largo de los cuerpos de agua, generar un buffer de 15 metros a cada lado (Dostal, 2007) destinados a la protección del recurso hídrico y a promover los corredores riparios en las rondas hídricas. 
- Dar prioridad a los predios que tengan presencia de pantanos, lagunas u otro tipo de cuerpos de agua lenticos y cobertura vegetal nativa o de transición al bosque, así como fauna silvestre que pueda tener afectado su hábitat.

- Considerar la permeabilidad y la capacidad de infiltración del suelo como un factor que contribuye a la regulación de los caudales y al mantenimiento de la oferta hídrica en los cauces, especialmente de aquellas formaciones geológicas que supongan la presencia de un acuífero.

- Identificar los predios o áreas susceptibles a fenómenos de remoción en masa, ya que sobre éstos se deberán tomar medidas de manejo de estabilización de taludes u otras estrategias que se consideren necesarias según sea el caso.

- Resaltar los predios que representen memoria histórica, sitios de interés cultural o puntos de referencia para la comunidad.

Toda la información recolectada deberá ser integrada y analizada, emitiendo una conclusión de las áreas escogidas, para facilitar esta labor, es necesario registrar o plasmar de manera objetiva y fácil de interpretar toda la información que ha sido procesada, para lo cual se recomienda la construcción de un modelo cartográfico que soporte y permita visualizar las diferentes áreas con sus respectivas características.

Para estudios detallados se propone la aplicación de la matriz descriptiva de cada área que reúne las principales variables a analizar y permite identificar cuáles de estas son prioritarias o estratégicas. (Véase Tabla 2. Matriz Descriptiva)

Dentro del análisis final, se definen predios con alta, moderada y baja importancia; lo cual sustenta la necesidad de conservación de cada área según sus características, como se evidencia en la Tabla 4, Tabla 5 y Tabla 6, respectivamente. Los predios o áreas con mayor importancia, serán considerados prioritarios.

\section{Predio de alta importancia}

Tabla 4. Características del predio clasificado de Alta Importancia

El predio presenta nacimientos de agua con presencia de drenajes a lo largo del mismo, cuenta además con la presencia de una gran zona pantanosa que contribuye a la regulación hídrica. Como se observa en la Figura 3. Se encuentra ubicado en medio de 3 unidades cartográfi-

AREA A cas de suelos; donde dos de estas son de páramo bajo que restringen el uso del suelo a cualquier actividad que no sea conservación o protección de los recursos.

Este predio es bien conocido por los habitantes de la cuenca, consideran que se debe proteger debido a la deforestación que se ha hecho.

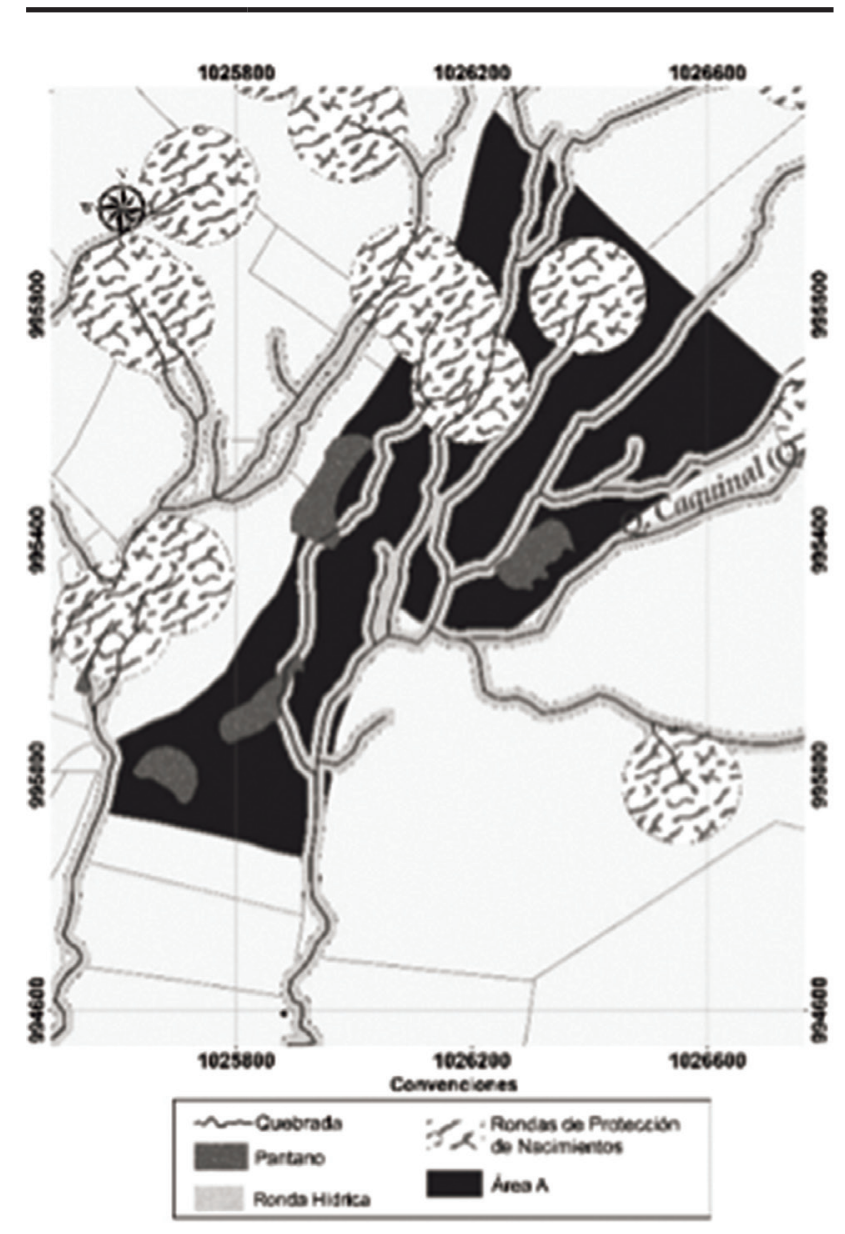

Figura 3. Predio de alta importancia 


\section{Predio de moderada importancia}

Tabla 5. Características del predio clasificado de Moderada Importancia

Se considera de moderada importancia por la presencia de un pantano el cual contribuye con la regulación hídrica; al no ser atravesado por cuerpos de agua no se le considera de importancia alta pero sí que tiene potencial para llegar a ser conser-

AREA B vado. Figura 4. Debido a la unidad de suelo presente en esta área, se recomienda la implementación de sistemas de potreros arbolados, ya que se puede llegar a originar procesos de remoción en masa, debido a la susceptibilidad que tienen estos a sufrir ese fenómeno.

\section{Predio de baja importancia}

Tabla 6. Características del predio clasificado de Baja Importancia

El predio no cuenta con cuerpos de agua que influyan en la regulación hídrica. Figura 5. Se ubica en una unidad de suelos que tiene capacidad para utilizarse en ganadería

AREA C extensiva con pastos naturales, asociada con actividades de agroforestería o para bosques protectoresproductores con labores de entresaca controladas o para regeneración espontánea de la vegetación.

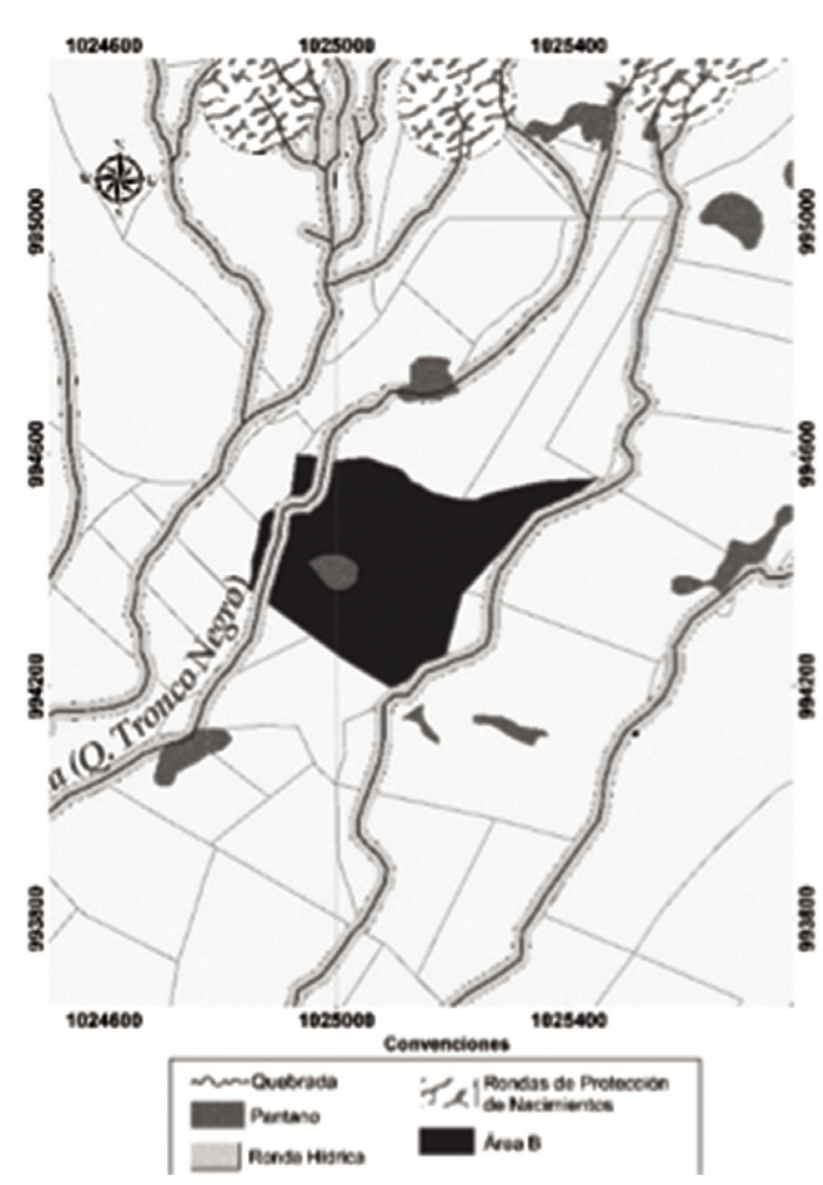

Figura 4. Predio de moderada importancia

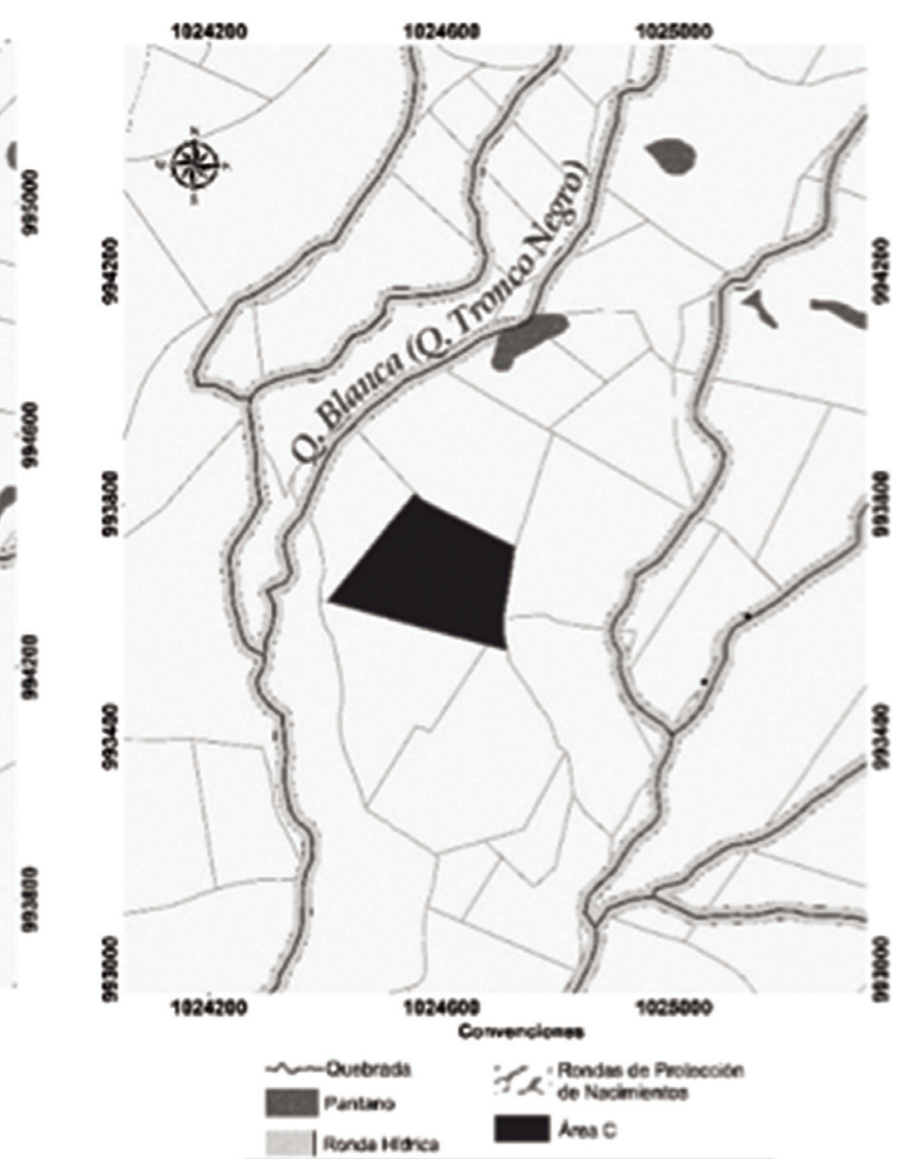

Figura 5. Predio de baja importancia 
Validación de la metodología en la microcuenca del río Caquinal

A partir de la aplicación de la metodología propuesta se definieron en total 21 áreas de especial importancia para la conservación y preservación del recurso hídrico, ubicadas en la cuenca alta del río Caquinal, estas suman 921,58 hectáreas, es decir que corresponden al $51,62 \%$ del área de la cuenca alta y al $18,46 \%$ del área total de la cuenca social. De los 21 predios, 7 son áreas protegidas y se encuentran en estado de rehabilitación natural, estas son propiedad de entidades gubernamentales 0 han sido declaradas reservas de la sociedad civil.

Es importante reconocer las potencialidades naturales que tiene el territorio como fortaleza en la recuperación y restauración de las áreas de recargas hídricas superficiales, que contribuirán en el mediano y largo plazo a la regulación de los caudales del río Caquinal. Las áreas naturales encontradas con alto valor ambiental son los pantanos, las rondas hídricas de cauces y los nacimientos de agua. Estas áreas contribuyen a mantener la conectividad en el sistema de cuenca y ayudan a mantener los niveles de agua en los cuerpos de agua gracias a la regulación hídrica otorgada por las características naturales que los caracterizan como el tipo de suelo, la vegetación y su capacidad de retención de agua.

Es necesario aunar el mayor número de esfuerzos técnicos y económicos para hacer frente a la dinámica que se viene presentando en la cuenca alta, para establecer posibles soluciones de amortización y mitigación de impactos que se hayan generado en los ecosistemas de regulación hídrica aguas arriba; así como trabajar en la prevención de futuras alteraciones no convenientes para el sistema natural, proceso que deberá ir de la mano con la concienciación y conocimiento de la comunidad de la región sobre la importancia de estas áreas para las generaciones venideras.

Será vital acordar con los propietarios de los predios, la definición de zonas de reforestación e incluso aplicar a incentivos económicos que se promueven desde organizaciones gubernamentales y no gubernamentales, para impulsar la sostenibilidad, la re-vegetalización y la protección de estas áreas.

Dada la naturaleza cualitativa de la metodología expuesta, no se incluyeron variables de orden cuantitativo, por lo cual el modelamiento de caudales y su comportamiento en el tiempo no hace parte del alcance de la investigación realizada.

\section{Discusión y Conclusiones}

La selección de áreas estratégicas, está relacionada con todos los elementos que componen una cuenca, por lo cual su evaluación no debe hacerse de manera apresurada y bajo un solo enfoque, sino que por el contrario debe incluir la mayor cantidad de variables posibles y objetivas que permitan reconocer las debilidades y fortalezas del sistema. La priorización de áreas a pesar de que se realiza en un lugar específico debe reconocer las características de toda la cuenca.

Estos procesos de priorización de áreas no sólo deben considerar las variables físico-ambientales, sino que también deben estar ligados a la memoria histórica y al valor que la comunidad le da a los lugares, como por ejemplo, dónde nace el agua que llega a sus predios, sabiendo que detrás de cada área existe todo un legado relacionado con las personas que han vivido o trabajado allí, elementos que no se pueden reconocer si no hay acercamiento a las comunidades y trabajo conjunto con ellas para la formulación y ejecución de un proyecto.

La importancia de priorizar áreas, en términos generales, puede clasificarse como de carácter técnico y de carácter social, siendo la primera más factible de cuantificar en términos físicos y funcionales, por ejemplo en pérdidas potenciales referidas a los daños que pueden sufrir los recursos 0 la interrupción de los servicios, a diferencia de la 
segunda, que prácticamente sólo puede valorarse cualitativamente y en forma relativa, debido a que está relacionado con aspectos económicos, educativos, culturales, ideológicos, etc.

La cuenca del río Caquinal cuenta con fortalezas sociales, ya que a través de los años la comunidad se ha organizado en torno a la gestión del recurso hídrico, sin embargo, será necesario tomar medidas serias frente a temas relacionados con el uso racional del agua y la aplicación de tecnologías apropiadas a los procesos productivos que se desarrollan en esta zona. Siendo importante fortalecer la articulación de la parte alta, media y baja de la cuenca, desde el propio reconocimiento, apropiación y capacidad asociativa en torno a la gestión del recurso hídrico. Por esta razón, fueron de gran ayuda todos los aportes realizados a esta investigación por parte de la comunidad, su apoyo e interés permanente por los resultados obtenidos.

Si bien la metodología propuesta se validó para un contexto en particular, se espera pueda ser utilizada en otros casos y con diferentes enfoques donde se busquen beneficios tanto para el sistema natural como para el sistema social, reconociendo que este último fortalece toda iniciativa que se quiera llevar a cabo. La metodología desarrollada constituye una herramienta para la toma de decisiones en la protección de cuencas hidrográficas.

\section{Literatura citada}

1. ASOCAQUINAL. (2010). Acta de constitución, aprobación de estatutos y elección de dignatarios y órganos de fiscalización. Foméque, Cundinamarca: Archivo ASOCAQUINAL.

2. Chow, V. T. (1994). Hidrología Aplicada. Mc Graw Hill.

3. CORPOGUAVIO. (2 de Diciembre de 2005). Diagnóstico y plan de ordenamiento y manejo de las áreas de drenaje de los ríos Blanco, Negro y Guatiquia en Fómeque y Río Blanco en Guasca, Fase de diagnóstico, prospectiva y formulación.

4. CORPOGUAVIO. (10 de Junio de 2011). Resolucion 345 de 2011.
5. Deeb, A. (1992). Estimación y cuantificación económica de algunas externalidades en proyectos de manejo de microcuencas. Banco Interamericano de desarrollo.

6. Dostal, C. B. (2007). Delimitación empírica de áreas prioritarias para el manejo del recurso hídrico en Costa Rica, 86(2), 39-49.

7. ESRI. (2010). ArcGIS Desktop. Redlands, California.

8. Estatutos ASOCAQUINAL. (2010). Foméque, Cundinamarca.

9. Goodman, L. A. (1961). Snowball Sampling. (I. f. Princeton University, Ed.) Journal Annals of Mathematics.

10. Gravelius, H. (1941). Flusskunde. Goschen'sche Verlagshandlung.

11. Gobernación de Cundinamarca. (2000). Mapa Base del Municipio de Fómeque

12. Gutiérrez-Malaxechebarría, A., \& Diaz-Granados, M. (2007). Valoración de Impactos y Evaluación Económica en Proyectos de Rehabilitación de Cuencas. Caso cuenca río Tona. Avances En Recursos Hidráulicos, (15), 3-10.

13. Horton, R. E. (1932). Drainage basin characteristics. Transactions of the American Geophysical Union(13), 350-361.

14. Horton, R. E. (1945). Erosional development of streams and their drainage basins; hydrophysical approach to quantitative morphology. Geological Society of America.

15. IGAC. (1995). Plancha Cartográfica 247-I-B-1 Escala 1:10.000.

16. Newton, A. y. (2011). Principios y práctica de la restauración del paisaje forestal: Estudios de caso en las zonas secas de América Latina. Union Internacional para la Conservación de la Naturaleza (UICN) y Madrid, España: Fundación Internacional para la Restauración de Ecosistemas, Gland, Suiza.

17. Restrepo, F. C. (2005). Valoración económica de ecosistemas estratégicos asociados a fuentes hídricas que abastecen acueductos veredales. Semestre Económico, 9.

18. Servicio Geológico Colombiano (SGC). (2000). Plancha geológica 247 escala 1:100.000.

19. Universidad Nacional de Colombia. (2000). Esquema de Ordenamiento Territorial. Municipios Jurisdicción CORPOGUAVIO. Técnico, Ciencias Económicas. Centro de Investigación para el desarrollo, Fómeque.

20. Zúñiga Palma, H. (2008). Hagamos el ordenamiento territorial del sector rural de nuestro municipio. Bogotá.

21. Zuñiga Palma, H. (2010). Valoración apropiada de la permeabilidad del suelo cualidad importante en la determinación de la erosionabilidad del territorio. Bogotá.

\section{Conflicto de Intereses}

Los autores declaran no tener ningún conflicto de intereses

Recibido: 8 de septiembre de 2014 Aceptado: 14 de octubre de 2014 